\title{
Ultrafast dynamics in the excited state of green fluorescent protein (wt) studied by frequency-resolved femtosecond pump-probe spectroscopy
}

\author{
Kathrin Winkler, Jörg Lindner, Vinod Subramaniam, Thomas M. Jovin \\ and Peter Vöhringer*
}

\author{
Max-Planck-Institute for Biophysical Chemistry, Biomolecular and Chemical Dynamics Group and \\ Department of Molecular Biology, Am Faßberg 11, Göttingen, 37077, Germany. \\ E-mail: pvoehri@gwdg.de
}

\author{
Received 1st October 2001, Accepted 13th December 2001 \\ First published as an Advance Article on the web 15th February 2002
}

\begin{abstract}
The time and frequency resolved optical response of wild-type green fluorescent protein (wt-GFP) has been measured at room temperature following $30 \mathrm{fs}, 400 \mathrm{~nm}$ photo-excitation. In the wavelength range covering the stationary fluorescence spectrum of the protein, the stimulated emission rises on a time scale of roughly $20 \mathrm{ps}$ due to excited-state proton-transfer (ESPT). The rise can be described phenomenologically by a sum of two exponentials. A long-time isosbestic behavior on the blue edge of the stationary emission implies a barrier for ESPT which is significantly larger than thermal excitations. In addition, an instantaneous component to the stimulated emission appears within the time resolution of our experiment. This observation is indicative of nonvertical cross-well transitions that prepare the proton-transferred configuration of the excited state directly from the equilibrium geometry of the ground-state neutral species during photo-excitation. Finally, transient absorptions around $500 \mathrm{~nm}$ and $650 \mathrm{~nm}$ can be observed, which are attributed to transitions from different protonated forms of the excited-state of GFP to higher lying electronic configurations, $\mathrm{S}_{n}$. The entire optical response of GFP is quantitatively simulated using a dynamic model that includes: (i) an energy-dependent rate coefficient for ESPT, (ii) intra- and intermolecular transfer of excess vibrational energy (IVR and VET), and (iii) an additional non-radiative decay pathway for the initially prepared Franck-Condon state leading to internal conversion via motion along a torsional coordinate. In particular, the nonexponential nature of the ESPT originates from overlapping time scales of reactive and non-reactive elementary processes following optical excitation.
\end{abstract}

\section{Introduction}

The photochemical and photophysical primary events leading to bioluminescence have recently attracted considerable interest. ${ }^{1}$ In particular, elucidation of the underlying microscopic molecular mechanisms and their time scales will help establish fundamental principles leading to photon emission from protein environments. Such information can then be used to custom design optical bio-chromophores with optimally adapted spectrochemical properties for a wealth of applications ranging from protein/membrane/DNA interactions to gene expression and non-invasive protein localization. ${ }^{1,2}$

Green fluorescent protein (GFP) of the jellyfish Aequoria victoria and site-specific mutations thereof have emerged as suitable prototypes for bioluminescent systems. Formation of the wild-type (wt) optical chromophore ( $p$-hydroxobenzylidene-imidazolidinone) occurs in the absence of co-factors via post-translational cyclization followed by autooxidation within a tripeptide unit of the polypeptide sequence consisting of 238 amino acids. ${ }^{3} \mathrm{X}$-ray diffraction has revealed a unique $\beta$ barrel tertiary structure in whose interior the chromophore is covalently anchored and thus effectively secluded from the aqueous solvent surrounding the protein. ${ }^{4,5}$ Additional noncovalent coupling of the chromophore to the protein backbone is facilitated via an extended hydrogen-bonded network that is believed to be of crucial importance to the optical response of the protein. ${ }^{6}$
The linear absorption spectrum of the protein consists of two resonances centered around $400 \mathrm{~nm}$ and $477 \mathrm{~nm}$. Excitation into the high energy band results in an intense green emission peaking at $508 \mathrm{~nm}$. This emission is extraordinarily stable to photo-bleaching ${ }^{3}$ as well as to variations of temperature, ${ }^{7}$ $\mathrm{pH}^{8}$, and ionic strength but vanishes in the presence of strong denaturants, 9,10 indicating that the $\beta$-barrel structural motif effectively prevents intramolecular non-radiative decay and/ or solvent-assisted quenching., ${ }^{7,9}$

Temperature-dependent fluorescence upconversion experiments including isotope substitution have led to a kinetic model in which both the ground and excited electronic states of GFP are characterized by two distinct conformations, A and $\mathrm{B}$, corresponding to the $400 \mathrm{~nm}$ and $477 \mathrm{~nm}$ absorptive resonances, which can interconvert by means of proton transfer. ${ }^{11}$ In the excited state, proton transfer from $\mathrm{A}^{*}$ occurs on a time scale of several picoseconds via barrier crossing to an intermediate, I*, which is responsible for the characteristic green emission. Presumably, I* constitutes an environmentally unrelaxed configuration of the proton-transferred form, $\mathrm{B}^{*}$, of the excited state. ${ }^{11}$

Complementary time-resolved emission studies on protein variants obtained by site-directed mutagenesis have been extremely helpful in eliciting possible pathways for proton transport from the optical chromophore to the backbone. ${ }^{12-16}$ Furthermore, low-temperature hole-burning studies on wt-GFP have proven highly instrumental not only in confirming the exis- 
tence of an intermediate but also in assigning the electronic origins ( $0-0$ transitions) associated with each of the three conformations. ${ }^{17,18}$ In the excited state, it appears as if $\mathrm{B}^{*}$ is separated from $\mathrm{I}^{*}$ by a barrier of about $2000 \mathrm{~cm}^{-1}$ whereas the barrier for $\mathrm{A}^{*}$ to $\mathrm{I}^{*}$ interconversion is somewhat lower. Likewise, in the ground state I must lie energetically above A and B, separated from the other forms by barriers whose heights are of the order of a few hundred wavenumbers. This explains the negligible population of I from cryogenic to room temperatures and allows for population trapping of I when prepared via the excited state at sufficiently low temperatures. ${ }^{17}$ Finally, it should be mentioned that GFPs have also been subject to numerous investigations at the single-molecule level including optical switching between conformations and blinking behavior. ${ }^{19-23}$ Also, most recent femtosecond pumpprobe experiments have revealed coherent wavepacket dynamics in the ground and excited states of the mutant EGFP, which exhibits only the red-shifted B-form absorption as well as improved brightness with respect to the wild-type protein. ${ }^{24}$

What remains to be explored in more detail are the time scales and the mechanisms associated with the elementary barrier crossing event ultimately leading to proton-transfer. This is because the excited-state proton-transfer (ESPT) kinetics were found to be highly multi-exponential, ${ }^{11,13}$ which is inconsistent with a simple excited-state interconversion between the neutral and anionic forms of the chromphore. According to Lossau et al., structural heterogeneities within the chromophore's microenvironment (e.g. an energetic and/or structural distribution of hydrogen-bonded systems) give rise to dispersive ESPT kinetics with a spectrum of time constants. ${ }^{13}$ Verifying such an interpretation must await single-molecule studies with sufficient time resolution to capture the primary ESPT event.

Here, we report femtosecond time and frequency resolved pump-probe studies of GFP with photoexcitation at $400 \mathrm{~nm}$. These efforts are aimed at quantifying the microscopic origin for dispersive kinetics as described above and unravelling the molecular mechanisms of the primary events involved in ESPT dynamics in GFPs. After some brief experimental details, our results will be presented in Section III. Two sets of experiments have been carried out, namely measurements of time-resolved pump-probe transients at a variety of fixed probe wavelengths ranging from $470 \mathrm{~nm}$ to $730 \mathrm{~nm}$ and measurements of timeresolved transient differential transmission spectra at a variety of fixed pump-probe time delays ranging from 0 ps to $100 \mathrm{ps}$. These two sets of experiments were designed to achieve the highest possible time resolution without sacrificing spectral resolution which, as we will see later, is highly beneficial for a quantitative analysis. The results will be discussed in Section IV with special emphasis on the primary protein/chromophore dynamics leading to bioluminescence.

\section{Experimental}

Femtosecond pump-probe measurements were carried out with a Ti:sapphire laser/regenerative chirped pulse amplifier system (CPA) capable of generating $800 \mathrm{~nm}, 30 \mathrm{fs}$ optical pulses with energies as high as $4 \mu \mathrm{J}$ and repetition rates of $250 \mathrm{kHz}$. A portion $(50 \%)$ of these amplified pulses was used to generate pump pulses centered at $400 \mathrm{~nm}$ by frequency doubling in a $0.2 \mathrm{~mm}$ thick Type-I BBO crystal with an efficiency of $15 \%$. Compensation for group-velocity dispersion (GVD) of the pump beam path was accomplished by using a pair of fused silica prisms and resulted in pulse durations of about $40 \mathrm{fs}$ at the location of the sample. The pump pulse energy was variably adjusted with a combination of a Glan-Taylor polarizer and a half-wave retardation plate.
The remaining $50 \%$ of the CPA output was split into two beams. The weaker one (20\%) served to generate a single-filament white-light continuum (WLC), which was used without further amplification for measurements of transient differential transmission spectra at well-defined pump-probe delays. To obtain tunable probe pulses, the WLC could also be amplified in a collinearly pumped optical parametric amplifier (OPA) which was driven by the second harmonic of the stronger beam. After GVD-compensation with a pair of $\mathrm{LAFN}_{28}$ prisms, nearly transform-limited probe pulses with durations of about $40 \mathrm{fs}$ and energies as high as $200 \mathrm{~nJ}$ were obtained throughout the entire visible spectral range between $470 \mathrm{~nm}$ and $700 \mathrm{~nm}$. A combination of a Glan-Taylor polarizer and a half-wave retardation plate was used to attenuate the tunable probe pulses to energies of about $1 \mathrm{~nJ}$.

Since photo-conversion can strongly modify the spectrotemporal response of GFP, the pump fluence and the total data acquisition time have to be minimized. In this respect, the rapid scan technique we described earlier is highly advantageous. ${ }^{25}$ Rapid scanning the pump-probe time delay was accomplished by steering the pump pulses through an optical assembly whose path length was dithered sinusoidally at a frequency of $10 \mathrm{~Hz}$ as described previously. ${ }^{25}$ Pump and probe pulses were spatially overlapped in the sample using achromatic lenses with $f=150 \mathrm{~mm}$. The pump and probe energies were typically reduced to $4 \mathrm{~nJ}$ and $1 \mathrm{~nJ}$, respectively. Probe pulse intensities were recorded behind and in front of the sample with standard Si-photodiodes whose output was directed to a fourth-order Butterworth filter with a $3 \mathrm{~dB}$ cutoff frequency of $50 \mathrm{kHz}$. The output of the analog filter was sent to a 16bit resolution digital oscilloscope (Tektronix, TDS 540) whose time base was triggered by an optical switch attached to the rapid scan delay line. In a total data acquisition time of $30 \mathrm{~s}$, a signal-to-noise ratio of better than 100 was easily achieved for pump-probe transients consisting of 5000 sampling points in a calibrated delay window of $23 \mathrm{ps}$.

For recording transient differential transmission spectra, the WLC, after being spatially and temporally overlapped in the sample, was dispersed in a $0.1 \mathrm{~m}$ polychromator (Bausch \& Lomb, resolution $1 \mathrm{~nm}$ ) and detected with a Si-photodiode array (Hamamatsu S3901-512Q). A computer controlled optical delay line (Melles Griot, Nanomover) was used to vary the pump-WLC probe time delay.

To further reduce signal perturbations due to photo-conversion, the sample consisted of a home-made optical cell capable of spinning around an axis perpendicular to the cell windows at a frequency of $5000 \mathrm{rpm}$. Recombinant wt-GFP as well as the mutant P4-3 were produced in Escherichia coli as described earlier. ${ }^{26}$ All measurements were performed at $\mathrm{pH} 7$. Photoconversion and/or photo-damage were regularly tested for during these measurements by recording UV/VIS absorption spectra of the sample after prolonged illumination by the pump and probe pulses. Under our experimental conditions, the spectra of the samples remained stable for several days unless the excitation energy was increased to above $10 \mathrm{~nJ}$ when rapid photo-conversion became evident as a gradual decrease of the relative absorbance $A(400 \mathrm{~nm}) / A(477 \mathrm{~nm})$.

\section{Results}

\section{A. Wavelength-dependent, time-resolved pump-probe transients}

Time resolved pump-probe transients with a total number of 36 different probe wavelengths ranging between 470 and 730 $\mathrm{nm}$ were measured. Between $470 \mathrm{~nm}$ and $630 \mathrm{~nm}$, the temporal evolution of the pump-probe signals is mostly governed by stimulation of the intense green emission characteristic for GFP. The time dependence in this wavelength range is compiled in 

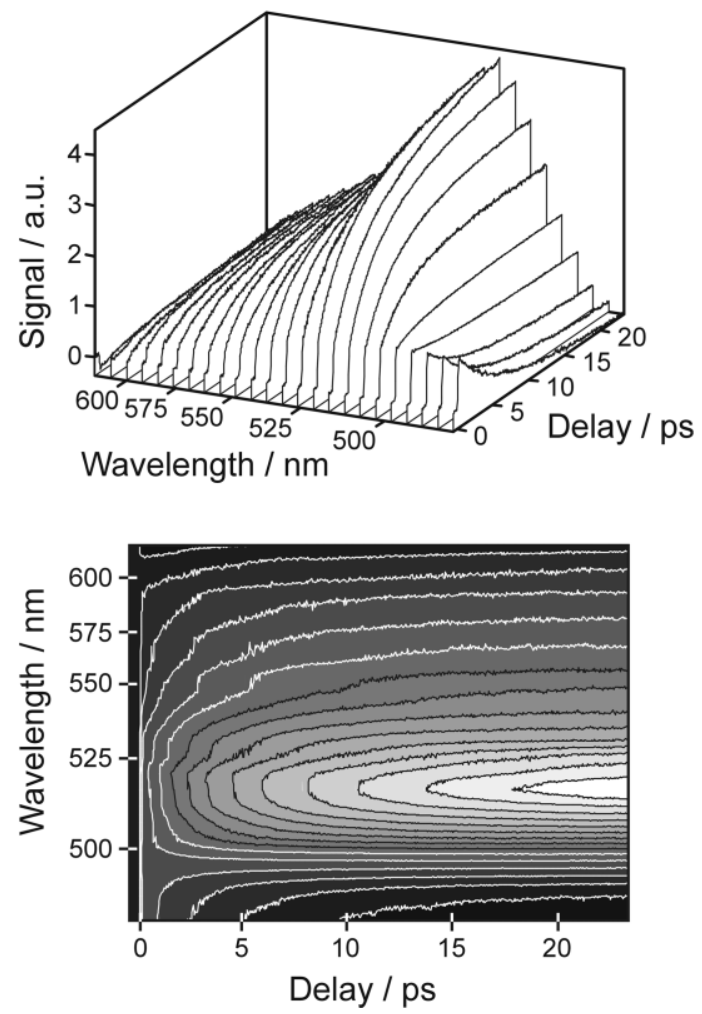

Fig. 1 Time-resolved pump-probe transients as a function of probe wavelengths in the region of the stationary emission spectrum of GFP. The excitation wavelength was $400 \mathrm{~nm}$. The lower panel displays a gray-shaded contour representation of the data.

Fig. 1. Representative transients on the blue edge, near the center, and on the red edge of the stationary emission spectrum of GFP are shown in Fig. 2. Qualitatively, for probe wavelengths longer than $496 \mathrm{~nm}$ and shorter than $600 \mathrm{~nm}$, the stimulated emission (e.g. gain) grows on a time scale of 20 ps. The data in this wavelength range can be fitted satisfactorily using a bi-exponential rise with time constants of roughly 2 ps and 8 ps. For wavelengths shorter than $496 \mathrm{~nm}$, this stimulated emission decays bi-exponentially with nearly the same time constants. A stimulated emission decay monitored at $494 \mathrm{~nm}$ exactly mirrors a stimulated emission growth at 498 $\mathrm{nm}$ except for delays shorter than roughly 0.5 ps (see Fig. 3). This behavior is indicative of an isosbestic point at an intermediate probe wavelength of around $496 \mathrm{~nm}$ and for delays larger than 0.5 ps. Furthermore, the pump-probe transients

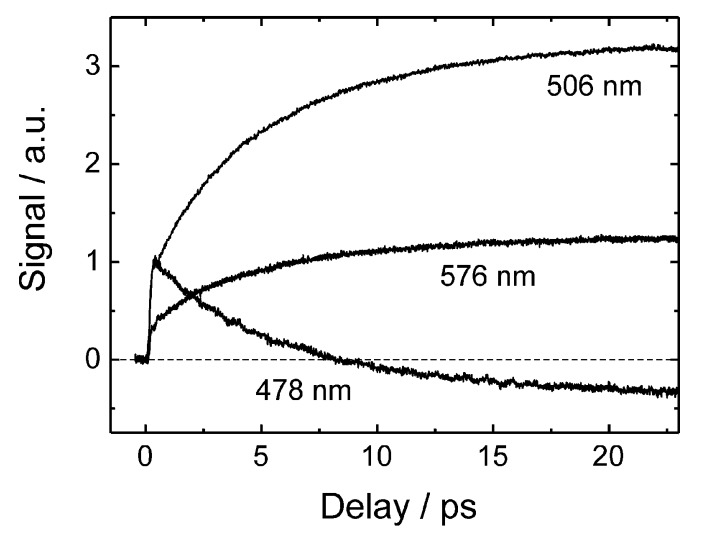

Fig. 2 Representative pump-probe transients at the blue edge (478 $\mathrm{nm})$, near the center $(506 \mathrm{~nm})$ and at the red edge $(576 \mathrm{~nm})$ of the steady-state fluorescence spectrum of GFP. Note the transient absorption at large pump-probe time delays for the short-wavelengths data.

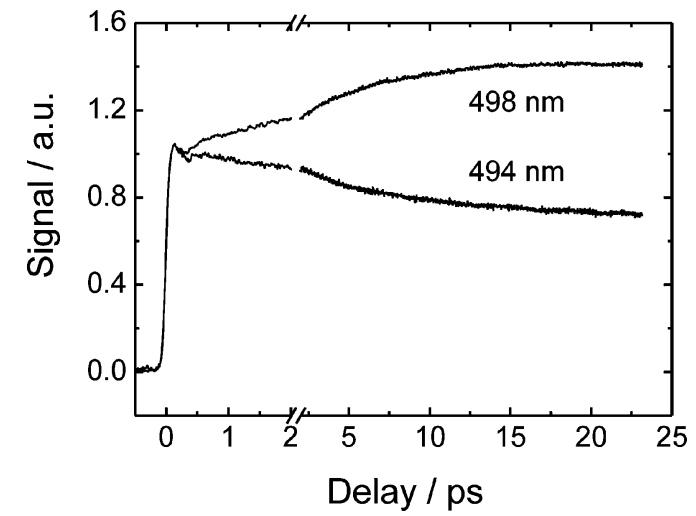

Fig. 3 Representative pump-probe transients at the blue edge of the steady-state fluorescence spectrum of GFP. The decay at $494 \mathrm{~nm}$ mirror images the rise at $498 \mathrm{~nm}$, indicating an isosbestic behavior for intermediate wavelengths.

recorded with the bluest probe wavelengths exhibit a long-time transient absorption into which the initial gain decays (see 478 $\mathrm{nm}$ transient of Fig. 2). Interestingly, for 470 $\mathrm{nm} \leq \lambda$ (Probe) $\leq 630 \mathrm{~nm}$, a stimulated emission can be detected immediately after photo-excitation within the timeresolution of our experimental setup. For wavelengths longer than $630 \mathrm{~nm}$, a distinct transient absorption can be observed, as emphasized in Fig. 4. It decays on a time scale similar to the growth of the gain observed around $508 \mathrm{~nm}$. However, an isosbestic point between $600 \mathrm{~nm}$ and $650 \mathrm{~nm}$ as reported by Michel-Beyerle and coworkers cannot be confirmed. ${ }^{13}$ Despite the signals being very weak in this wavelength range, a pronounced dynamic evolution can nevertheless be observed as shown in Fig. 4. Furthermore, under our excitation conditions, there is no evidence for an additional ultrafast (700 fs) decay of the stimulated emission near $580 \mathrm{~nm}$ as reported in ref. 13 (see also Fig. 2). Finally, we note that, below 1 ps, coherent wavepacket dynamics of the protein can be detected as oscillatory signal modulations in both stimulated emission and transient absorption regions.

\section{B. Time-dependent differential transmission spectra}

In order to verify an isosbestic point in the vicinity of $496 \mathrm{~nm}$ and to spectrally characterize the transient absorption observed in the blue at long pump-probe time delays, timedependent differential transmission spectra of GFP were recorded following photo-excitation at $400 \mathrm{~nm}$. Such spectra also allow for a proper normalization of the pump-probe transients described in the previous section.

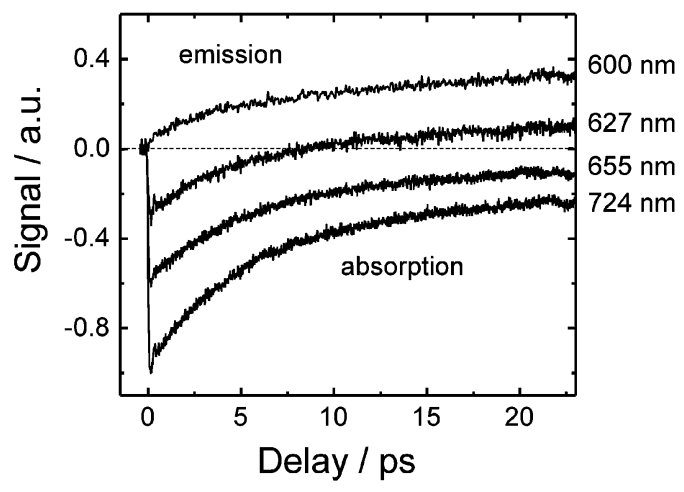

Fig. 4 Representative pump-probe transients at the far red edge of the steady-state fluorescence spectrum of GFP. For wavelengths longer than $630 \mathrm{~nm}$, the signals are dominated by a transient absorption from $\mathrm{A}^{*}$. 
Representative spectra between $470 \mathrm{~nm}$ and $600 \mathrm{~nm}$ are displayed in Fig. 5 for various pump-probe time delays. There are several aspects which are worth highlighting:

(i) At the shortest pump-probe time delays, the stimulated emission is much broader than the stationary fluorescence spectrum of GFP.

(ii) Within the signal-to-noise ratio, an isosbestic point can indeed be observed at a wavelength of $496 \mathrm{~nm}$.

(iii) After about $7 \mathrm{ps}$, the red edge of the differential transmission spectrum matches the spontaneous fluorescence spectrum of GFP quantitatively.

(iv) Within the maximum pump-probe time delay, no spectral shift of the stimulated emission is discernible excluding an environmental reorganization around the chromophore similar to solvation.

(v) The shoulder reminiscent of the vibrational substructure of the GFP fluorescence is visible at the earliest delays but it becomes more pronounced with increasing pump-probe time delay.

(vi) A residual long-time transient absorption is again observed for wavelengths shorter than $480 \mathrm{~nm}$.

The quantitative agreement between spontaneous emission and long-time transient gain for wavelengths longer than 508 $\mathrm{nm}$ can be exploited to extract the spectral behavior of the transient absorption observed in the blue. Subtracting the stationary fluorescence spectrum of GFP from the transient differential transmission spectrum recorded at a time delay of $100 \mathrm{ps}$ results in an absorption spectrum which is reproduced in the lower panel of Fig. 5. This spectrum peaks at roughly $500 \mathrm{~nm}$ and exhibits a shoulder around $480 \mathrm{~nm}$ indicating additional contributions from a Franck-Condon active mode with a frequency of approximately $830 \mathrm{~cm}^{-1}$.

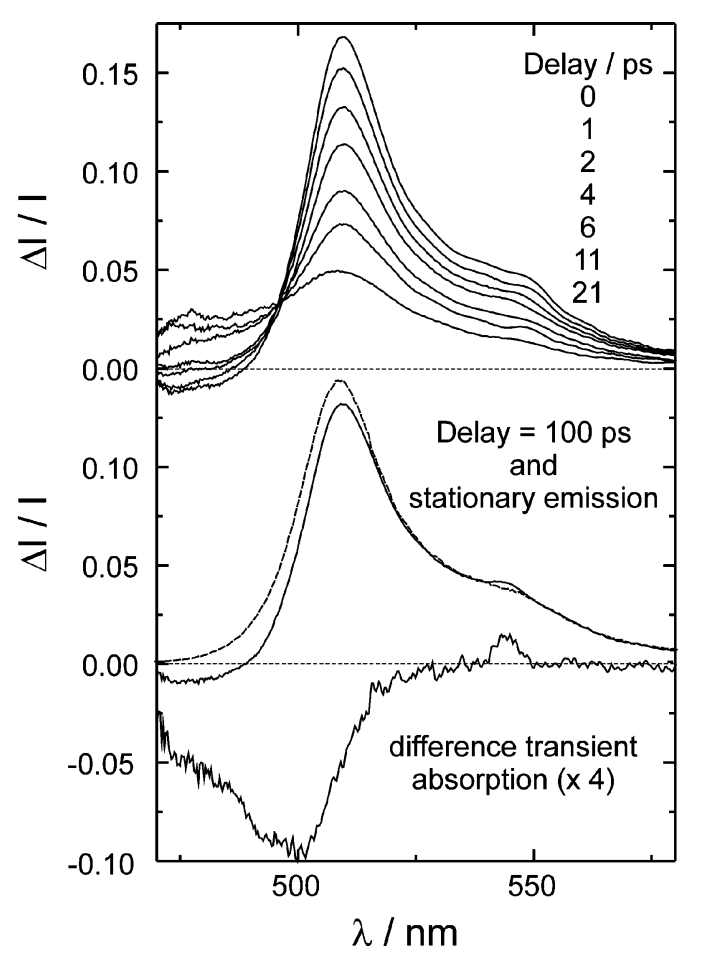

Fig. 5 Upper panel: Transient differential transmission spectra of GFP following optical excitation at $400 \mathrm{~nm}$. Note the isosbestic point at $496 \mathrm{~nm}$ and the long-time residual transient absorption in the blue. Also, the shoulder around $550 \mathrm{~nm}$ is visible even at the earliest pumpprobe time delay, indicating that the spectrum around 0 ps already reflects a mixture of the neutral and deprotonated form of the excited state. Lower panel: Comparison between the steady-state (dashed) and the transient spectrum (solid) of GFP at $100 \mathrm{ps}$. The difference yields a transient absorption centered at $500 \mathrm{~nm}$.
The transient absorption can be represented adequately by a sum of two Gaussian profiles

$$
\varepsilon_{\mathrm{TA}}(\lambda)=-\sum_{i} \varepsilon_{i} \exp \left(-4 \ln (2) \frac{\left(\varepsilon-\varepsilon_{i}\right)^{2}}{\Delta \varepsilon_{i}}\right)
$$

whose center wavelengths, $\lambda_{i}$, are 499 and $479 \mathrm{~nm}$. Their corresponding relative amplitudes, $\varepsilon_{i}$, are 1 and 0.55 , respectively. Both Gaussians have the same full spectral width at half maximum of $\Delta \lambda_{i}=20 \mathrm{~nm}$. For later use, we also decompose the stationary emission spectrum of GFP into a sum of Kubo lineshapes $^{27}$

$$
\begin{aligned}
\varepsilon_{\mathrm{E}}(\omega)= & \int_{-\infty}^{\infty} \mathrm{d} t \mathrm{e}^{\mathrm{i} \omega t} \sum_{i} \varepsilon_{i}\left\{\frac{\Delta^{2}}{\gamma}\left(1-\mathrm{e}^{-\gamma t}\right) \cos \left(\Omega_{i} t\right)+\Omega_{i} \sin \left(\Omega_{i} t\right)\right\} \\
& \times \exp \left[-\frac{\Delta^{2}}{\gamma^{2}}\left(\mathrm{e}^{-\gamma t}+\gamma t-1\right)\right]
\end{aligned}
$$

where $\omega=2 \pi c / \lambda$ and $c$ is the speed of light. Their amplitudes, $\varepsilon_{i}$, are $0.89,0.15,0.12,0.17$, and 0.05 and their corresponding center wavelengths, $\lambda_{i}=2 \pi c / \Omega_{i}$, are 508.6, 523.2, 533.9, 547.6, and $570 \mathrm{~nm}$. Each component is broadened equally by setting $\Delta=\gamma=490 \mathrm{~cm}^{-1}$. Fitting the spectra to these peculiar functional forms has no physical rationale and is only intended to provide a numerically convenient representation of the data.

\section{Discussion}

\section{A. Background}

From low-temperature hole-burning studies, a rather detailed energy level scheme for GFP has been proposed by Creemers et $a l .{ }^{17}$ which can be tested against the time and frequency resolved data presented here. The energy-level diagram is qualitatively reproduced in Fig. 6. First, it should be mentioned that an excitation wavelength of $400 \mathrm{~nm}$ prepares GFP in its excited state with an excess energy of about $2000 \mathrm{~cm}^{-1}$ with respect to a vertical $0-0$ transition from $A$ or with an excess

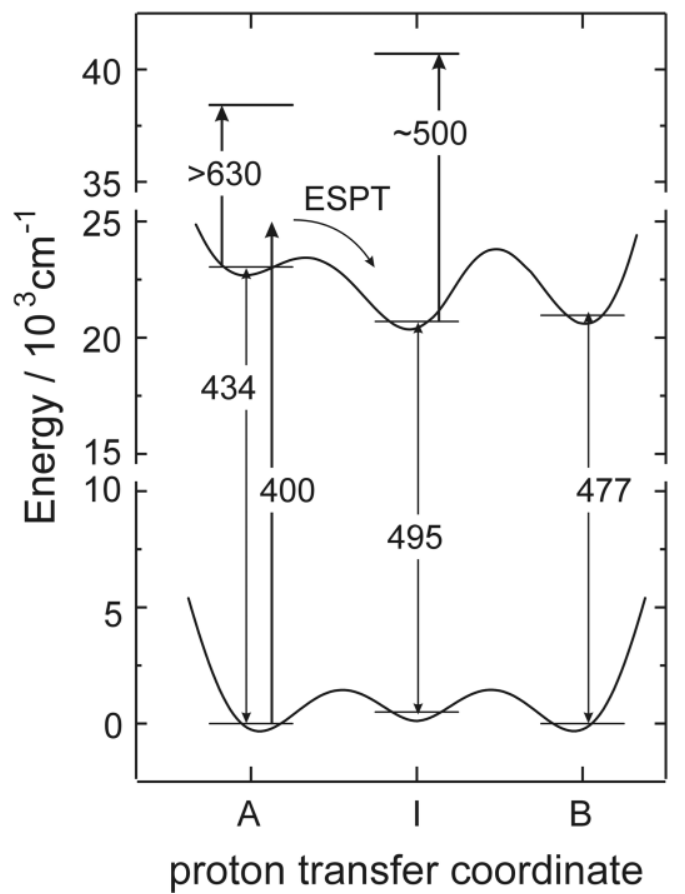

Fig. 6 Energetic scheme of the three different photoconvertible forms A, I, and B of wt-GFP. Double-headed arrows indicate their $0-0$ transitions in $\mathrm{nm} .{ }^{17}$ Also included are the two transient absorptions to higher lying electronic configurations of GFP observed in this work. 
energy of roughly $5000 \mathrm{~cm}^{-1}$ with respect to vertical $0-0$ transitions from I or B, respectively. This excess energy lies above the barrier for an interconversion between $\mathrm{B}^{*}$ and $\mathrm{I}^{*}$ which amounts to roughly $2000 \mathrm{~cm}^{-1}$. Since ground-state absorptions from A and B are still partially overlapped at $400 \mathrm{~nm}$, direct photo-excitation of $\mathrm{B}$ has to be taken into account. Using an energy difference of the ground state species A and $\mathrm{B}$ of less than $2 k_{\mathrm{B}} T$ in accordance with their relative occupancies, ${ }^{6}$ the $0-0$ transitions for $\mathrm{A} \rightarrow \mathrm{A}^{*}$ and $\mathrm{B} \rightarrow \mathrm{B}^{*}$ imply an energy gap between $A^{*}$ and $B^{*}$ of roughly $2000 \mathrm{~cm}^{-1}$. Thus direct photo-conversion from $\mathrm{A}^{*}$ to $\mathrm{B}^{*}$ is energetically allowed provided the barrier for $I^{*}$-formation from $\mathrm{A}^{*}$ is lower than from $\mathrm{B}^{*}$ as indeed suggested by the hole burning studies. ${ }^{17}$ One can estimate a lower limit for the barrier between $\mathrm{A}^{*}$ to $I^{*}$ from the temperature dependent fluorescence upconversion experiments performed by the Boxer group. ${ }^{11}$ According to their data, the fluorescence from $\mathrm{A}^{*}$ is found in the region between 420 and $470 \mathrm{~nm}$ (i.e. on the blue side of Fig. 5). At $85 \mathrm{~K}$, this emission does not decay at all on a time scale of 30 ps whereas at room temperature its lifetime is roughly 4 ps as judged by the fastest time constant of a triple-exponential decay. ${ }^{11}$ Hence, there must be a barrier which is at least of the order of $k_{\mathrm{B}} T$ at room temperature, i.e. $\geq 200 \mathrm{~cm}^{-1}$. Such a barrier would be in accord with our finding of an isosbestic point at $496 \mathrm{~nm}$, implying a simple 2-state interconversion process with exponential-type relaxation dynamics as well as shape stability of the absorptive and emissive resonances of all the species involved.

In terms of the model of refs. 11 and 17, the build-up of the emission centered at $508 \mathrm{~nm}$ is undoubtedly due to formation of I* (see Fig. 2) whereas the decay of the transient absorption in the near infrared is due to the decay of $A^{*}$ (see Fig. 4). Since the transient absorption centered at $500 \mathrm{~nm}$ persists on time scales of 100 ps (see Fig. 5), it must be due to I*. Both of these absorptive transitions to higher lying electronic configurations of GFP are indicated in Fig. 6 by vertical arrows. However, the origin of the early-time (i.e. prompt) stimulated emission remains unclear. In addition, the relaxation dynamics are not truly single exponential and the chromophore is initially vibrationally excited.

\section{B. Prompt emission and cross-well excitation}

According to Boxer and coworkers, ${ }^{11}$ prompt fluorescence results from direct excitation from $\mathrm{B}^{\text {to }} \mathrm{B}^{*}$, which is believed to emit at center wavelengths intermediate to the $\mathrm{A}^{*}$ and $\mathrm{I}^{*}$ fluorescence.

To elucidate possible contributions from $\mathrm{B}^{*}$, photo-conversion experiments were performed. By extended long-time exposure to $20 \mathrm{~mW}$ of our $400 \mathrm{~nm}$ femtosecond pulses, the samples could be substantially photo-converted to the B-form, as judged by inverted A and B absorbances in the UV/VIS absorption spectrum. However, increasing the population of $\mathrm{B}$ by a factor of five did not change the relative amplitudes of prompt (i.e. early-time) and delayed emission at a probe wavelength of $530 \mathrm{~nm}$ by more than $10 \%$. Therefore, under our excitation conditions, $\mathrm{B}^{*}$ cannot be held responsible for causing the prompt emission.

Hence, one might conclude that this prompt emission corresponds to the low-energy tail of the A* fluorescence. Its decay should be the mirror image of the rise of the I* emission as is indeed observed in Fig. 3. The isosbestic point was then located at a wavelength where the cross section for stimulated emission from $\mathrm{A}^{*}$ equals the cross section for stimulated emission from I*. Such an interpretation, however, requires the $\mathrm{A}^{*}$ fluorescence to be peaked at $508 \mathrm{~nm}$ (see Fig. 5) and to be extremely broad, spanning a wavelength range from $400 \mathrm{~nm}$ to well beyond $600 \mathrm{~nm}$ where it is still clearly visible within our signal-to-noise ratio. A spectral width of more than 8000 $\mathrm{cm}^{-1}$ for the $A^{*}$ fluorescence seems unrealistic in the light of the $3800 \mathrm{~cm}^{-1}$ spectral width of the corresponding groundstate absorption from A. ${ }^{11}$

Instead, we believe that the prompt broad emission already represents a mixture of $\mathrm{A}^{*}$ and $\mathrm{I}^{*}$ fluorescence. For wavelengths shorter than the isosbestic wavelength, the gain is dominated by $\mathrm{A}^{*}$ whereas the gain seen at longer wavelengths is primarily due to $I^{*}$. This interpretation is supported by the weak shoulder around $550 \mathrm{~nm}$ which is clearly reminiscent of the I* fluorescence but already observable even at the earliest pump-probe time delays (see Fig. 5). Light-induced preparation of $\mathrm{I}^{*}$ must occur either via direct excitation of I or via non-vertical cross-well excitation directly from the groundstate A-form. Since I is not populated at cryogenic temperatures and only weakly populated at room temperature according to the hole-burning studies, it follows that cross-well transitions from A are likely to be the dominant mechanism for the prompt appearance of $I^{*}$ emission. Such cross-well excitations become possible due to non-vanishing FranckCondon factors between the vibrational eigenfunctions of the ground-state A-form and those of the excited-state I-form. Nonvertical transitions have also been studied experimentally ${ }^{28}$ as well as theoretically ${ }^{29}$ on intramolecular ESPT systems of smaller size.

\section{Failure of the simple 2-state interconversion scheme}

We now turn our attention to the isosbestic behavior indicative of a barrier between $A^{*}$ and $I^{*}$. In the absence of a barrier, the relaxation from $\mathrm{A}^{*}$ to $\mathrm{I}^{*}$ would result in a complex dynamic spectral shifting as the population drifts downhill towards the intermediate. At a first glance, the observation of an isosbestic point implies a primary equilibration in the $\mathrm{A}^{*}$ well which is faster than the barrier crossing event itself. Note that the simplified energy level scheme depicted in Fig. 6 represents a one-dimensional cross-section (preferentially along the proton-transfer reaction coordinate) through a multi-dimensional hypersurface of the excited state of GFP. Considering the $3 N-6$ vibrational modes of the chromophore only, this dimensionality is at least 50 . For a molecule with such a high number of vibrational degrees of freedom, an excess energy of $2000 \mathrm{~cm}^{-1}$ with respect to $A^{*}$ may not necessarily correspond to a high degree of vibrational excitation, particularly with respect to the high-frequency modes of the chromophore. This is because rapid intramolecular vibrational redistribution (IVR) may lead to efficient flow of excess vibrational energy from the few Franck-Condon active modes of the chromophore to other so-called dark modes whose Franck-Condon factors are negligible. Even though the mean excess energy calculated over all $3 N-6$ vibrational degrees of freedom still amounts to $2000 \mathrm{~cm}^{-1}$, high-frequency modes such as $\mathrm{OH}$ and $\mathrm{NH}$-stretches which are likely to couple to the reaction coordinate may then be vibrationally relaxed. Hence, provided IVR is complete in those modes which closely resemble the reaction coordinate, the chromophore might very well be equilibrated with respect to the one-dimensional $\mathrm{A}^{*}$ well as depicted in Fig. 6. If, in addition, these degrees of freedom are the principal Franck-Condon active modes of the chromophore, emission from $A^{*}$ becomes rather insensitive to the amount of excess energy initially deposited upon optical excitation. At any rate, vibrational energy transfer (VET) from the chromophore to its surroundings will ultimately facilitate a true dissipation of the $2000 \mathrm{~cm}^{-1}$ excess energy.

Once IVR and VET have led to vibrational thermalization in both the $\mathrm{A}^{*}$ and the $\mathrm{I}^{*}$ wells, frequent barrier crossings and re-crossings concurrent with forward and backward ESPT will establish equilibrium between the $\mathrm{A}^{*}$ and $\mathrm{I}^{*}$ populations followed by non-radiative decay to the ground state on nanosecond time scales. Provided energy-specific rate constants, $k(E)$, for the barrier crossing event from $\mathrm{A}^{*}$ to $\mathrm{I}^{*}$ are known either through experiment or calculated using existing unimo- 
lecular rate theories, the thermal rate coefficient for ESPT, $k(T)$, can be evaluated by averaging $k(E)$ over the temperature-dependent but stationary vibrational distribution within the reactant well. Such a scenario fully explains the existence of an isosbestic point observed at $496 \mathrm{~nm}$ but fails to explain the non-exponential nature of the observed kinetics.

At this stage, it is instructive to investigate the non-exponentiality in more detail. Dispersive ESPT kinetics with multiexponential decays/growths have also been observed in timeresolved fluorescence data of wt-GFP by Michel-Beyerle, Boxer and their coworkers. ${ }^{11,13}$ More quantitatively, it was shown that the I* emission grows bi-exponentially with time constants of roughly 2 and 8 ps in agreement with the data presented here. ${ }^{11,13}$ Both time constants exhibit a pronounced kinetic isotope effect, clearly indicating that this time scale is indeed governed by proton transfer. ${ }^{11,13}$ Lossau. et al. suggested that the non-exponential behavior can be traced back to structural and energetic heterogeneities of the hydrogenbonded network in which the chromophore is embedded. Unfavorable structures give rise to rather long time constants which become increasingly important as the temperature is lowered. ${ }^{13}$

However, according to refs. 13 and 16, the fluorescence decay of $\mathrm{A}^{*}$ measured with a streak camera and a time resolution of 6 ps contains an additional exponential component with a time constant well in excess of 200 ps. A similar longtime decay of $\mathrm{A}^{*}$ with a time constant of $120 \mathrm{ps}$ is also observed in femtosecond fluorescence upconversion experiments. ${ }^{11}$ Interestingly, such a component does not have a rising counterpart when $I^{*}$ emission is monitored. ${ }^{11,13,16}$ This lack of correlation between $\mathrm{A}^{*}$ and $\mathrm{I}^{*}$ fluorescence at long delay times is inconsistent with a distribution of ESPT time constants due to structural heterogeneities. Rather, it indicates that the long time decay of $\mathrm{A}^{*}$ is not directly linked to ESPT dynamics. In fact, deuteration does not influence the time constant of this component at all, ${ }^{11,13,16}$ thereby lending further credence to the idea that the tail of the $\mathrm{A}^{*}$ decay reflects additional deactivation pathways other than proton transfer.

Kummer et al. studied the dynamics of internal conversion in the wild-type protein as well as in a number of different site-specific mutants. ${ }^{15}$ They found that this second radiationless decay channel has an average quantum yield of roughly $10-25 \%$ and also exhibits a pronounced non-exponentiality. More quantitatively, a mean time constant for internal conversion of $\mathrm{A}^{*}$ in the wild-type protein of 150 ps was derived. Although this time constant decreased to only 10 ps upon the substitution Phe64Leu, Ser65Thr, and Thr203Tyr, the ESPT channel was still found to be fast enough to significantly compete with internal conversion. ${ }^{15}$

It was concluded that internal conversion requires motion along torsional degrees of freedom of the chromophore, which, in turn, can be expected to be sensitive to finer detail regarding the microenvironments (such as e.g. rigidity). ${ }^{15,30}$ This effect can be understood in terms of an effective friction which hinders motion along the torsional coordinate, similar to diffusive barrier crossing dynamics involved in large amplitude isomerization reactions. Similarly to ESPT, however, such a nonradiative decay pathway should also be affected by IVR and VET processes as discussed above. Hence, internal conversion can be held responsible neither for the mismatch of the longtime tails seen in I* and $\mathrm{A}^{*}$ emission nor for the non-exponential kinetics unless IVR and VET are not truly de-coupled from the elementary barrier crossing event leading to ESPT.

\section{Refined dynamic model for ESPT}

If indeed ESPT barrier crossing dynamics and non-reactive IVR/VET processes occur on similar time scales, the thermal rate coefficient for ESPT must exhibit a pronounced timedependence.
Immediately after photo-excitation but before IVR and VET processes have fully been brought into action, the chromophores are still dressed with an average excess energy in the reactive modes which lies substantially above the barrier for $\mathrm{A}^{*}$ to I* interconversion. Subsequently, IVR and VET processes smoothly transfer vibrational excitation from the reactive modes to either non-reactive degrees of freedom of the chromophore or to its environment while simultaneously transforming the initially microcanonical distribution well above the barrier for ESPT into a canonical distribution that is primarily located below the threshold for proton transfer. Consequently, a time dependent rate coefficient, $\mathrm{k}(T, t)$, can be expected that essentially tracks the dynamically evolving distribution within the reactive vibrational modes of the chromophore via their intrinsic energy-specific rate constants, $k(E)$. Hence, in order to obtain a meaningful rate coefficient for ESPT, an averaging of energy-specific rate constants has to be performed over an instantaneous (i.e. time-dependent) microcanonical distribution. Only in the long-time limit, is the true thermal rate constant recovered as the well-known canonical average over energy-specific rate constants.

Even though our data do not provide conclusive evidence for a time-dependent rate coefficient for ESPT, the above discussion demonstrates that non-reactive inter- and intramolecular chromophore dynamics have to be taken into account when GFP is excited above the barrier for $\mathrm{A}^{*}$ to I* interconversion. Note that most of the existing spectroscopic studies on wtGFP published so far have been performed at an excitation wavelength of $400 \mathrm{~nm}$, corresponding to $2000 \mathrm{~cm}^{-1}$ of excess energy in $\mathrm{A}^{*}$. This also holds for recent lifetime measurements using 2-photon excitation with $800 \mathrm{~nm}$ light. ${ }^{31,32}$

Further complications may arise from the energy-specific rate constants for torsional barrier crossing leading to internal conversion having a different energy dependence as compared to those describing ESPT barrier crossing. Under such circumstances, channel switching might become important during the radiationless decay which would then explain the tail mismatch between $I^{*}$ and $\mathrm{A}^{*}$ emissions as mentioned earlier.

The following scenario might be envisioned in which the chromophore is excited with $400 \mathrm{~nm}$ well above both the torsional and ESPT barriers. At early times, before equilibration in the $\mathrm{A}^{*}$ well is complete, ESPT is the dominant decay channel for $\mathrm{A}^{*}$. As IVR and VET progressively withdraw excess vibrational energy from the reactive modes, ESPT gradually decelerates while torsional barrier crossing with subsequent internal conversion to A becomes increasingly important. At infinite times, the chromophores are located below the threshold for ESPT, leaving internal conversion as the dominant pathway for radiationless deactivation of $\mathrm{A}^{*}$. Consequently, at early times, the rise of the $I^{*}$ emission is correlated with the decay of the $\mathrm{A}^{*}$ emission. However at later times, $\mathrm{A}^{*}$ decays predominantly via internal conversion. This would explain the non-exponential nature of the observed dynamics, the pronounced deuteration dependence of the fast components (seen in emission from both $\mathrm{A}^{*}$ and $\left.I^{*}\right)$, the lack of a kinetic isotope effect for the slow components (seen only in emission from $\mathrm{A}^{*}$ ), and most importantly, the distinctly different behavior of $\mathrm{A}^{*}$ and $\mathrm{I}^{*}$ on time scales above 100 ps.

To quantify these concepts, a kinetic model can be outlined that captures the essential dynamics (see Fig. 7). The species $\mathrm{A}_{\mathrm{h}}^{*}, \mathrm{~A}_{\mathrm{p}}^{*}$, and $\mathrm{A}_{\mathrm{c}}^{*}$ refer to the hot, the partially relaxed, and the fully equilibrated A-form with respect to vibrational excitation in the reactive modes. The species $\mathrm{A}_{\mathrm{h}}^{*}$ and $\mathrm{A}_{\mathrm{p}}^{*}$ can undergo proton transfer to the I-form with energy-specific rate coefficients $k_{\mathrm{h}}$ and $k_{\mathrm{p}}$, respectively. This channel competes with internal conversion via torsional barrier crossing which is described by the rate-coefficient, $k_{\mathrm{T}}$. For simplicity, the latter is taken to be energy independent. Once fully equilibrated, the only channel which remains open is torsion. 


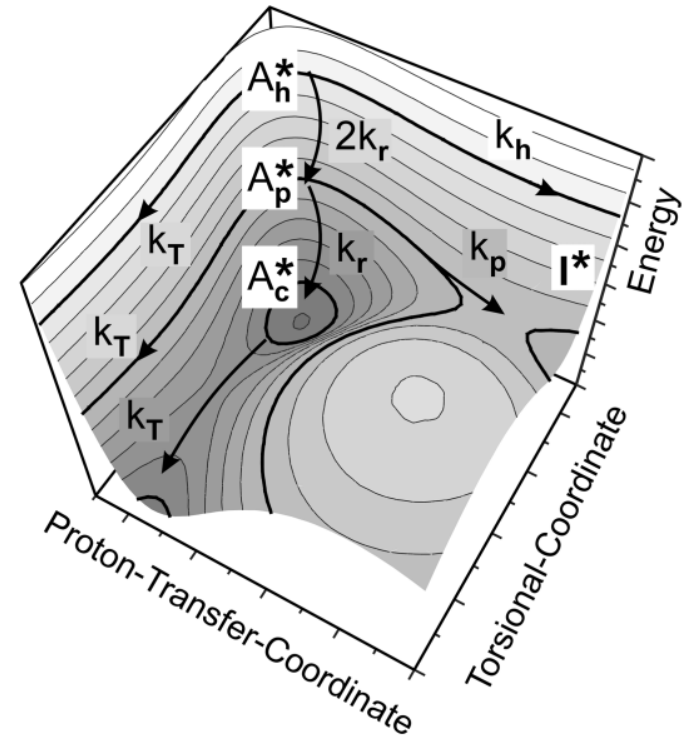

Fig. 7 Refined dynamic model for the excited-state proton transfer of wt-GFP. $A_{h}^{*}, A_{p}^{*}$, and $A_{c}^{*}$ denote the vibrationally excited, the partially relaxed, and the fully relaxed state of the electronically excited neutral chromophore. The rate constant for torsional motion required for subsequent internal conversion is given by $k_{\mathrm{T}}$. Vibrational relaxation is treated in the harmonic approximation and is specified by the rate constant $\mathrm{k}_{\mathrm{r}}$. The rate constant for ESPT is energy-dependent such that $\mathrm{A}_{\mathrm{h}}^{*}$ transfers a proton with rate constant $k_{\mathrm{h}}$ and $\mathrm{A}_{\mathrm{p}}^{*}$ ionizes with rate constant $k_{\mathrm{p}}$. Finally, once the neutral chromophore is vibrationally cold, the ESPT channel is closed and $k_{\mathrm{c}}=0$.

Energy relaxation is described in the harmonic approximation by setting the rate for transitions from $A_{h}^{*}$ to $A_{p}^{*}$ to twice the rate for transitions occurring from $A_{p}^{*}$ to $A_{c}^{*}$. Note, that we do not invoke detailed balancing to simplify the calculation. Hence, since the excess energy is roughly $2000 \mathrm{~cm}^{-1}$ with respect to the vibrationless level in $A^{*}$, the species $A_{h}^{*}$ and $\mathrm{A}_{\mathrm{p}}^{*}$ correspond to 2 and 1 quanta of excitation, respectively, in a fictitious vibrational mode with a frequency of 1000 $\mathrm{cm}^{-1}$. Obviously, finer energy graining with respect to $A^{*}$ provides a more realistic description of the vibrational dynamics, but at the expense of losing an analytic solution to the kinetic differential equations. Referring to Fig. 7 and assuming $\left[\mathrm{A}_{\mathrm{p}}^{*}(0)\right]=\left[\mathrm{A}_{\mathrm{c}}^{*}(0)\right]=0$, the time dependent populations can be derived

$$
\begin{gathered}
\frac{\left[\mathrm{A}_{\mathrm{h}}^{*}(t)\right]}{\left[\mathrm{A}_{\mathrm{h}}^{*}(0)\right]}=\mathrm{e}^{-k_{\gamma} t} \\
\frac{\left[\mathrm{A}_{\mathrm{p}}^{*}(t)\right]}{\left[\mathrm{A}_{\mathrm{h}}^{*}(0)\right]}=\frac{2 k_{\mathrm{r}}}{k_{\beta}}\left\{\mathrm{e}^{-k_{\gamma} t}-\mathrm{e}^{-k_{\alpha} t}\right\} \\
\frac{\left[\mathrm{A}_{\mathrm{c}}^{*}(t)\right]}{\left[\mathrm{A}_{\mathrm{h}}^{*}(0)\right]}=2 k_{\mathrm{r}}^{2}\left\{\frac{1}{k_{\beta}\left(k_{\mathrm{r}}+k_{\mathrm{p}}\right)} \mathrm{e}^{-k_{\alpha} t}\right. \\
\left.+\frac{1}{\left(2 k_{\mathrm{r}}+k_{\mathrm{h}}\right)\left(k_{\mathrm{r}}+k_{\mathrm{p}}\right)} \mathrm{e}^{-k_{\mathrm{T}} t}-\frac{1}{k_{\beta}\left(2 k_{\mathrm{r}}+k_{\mathrm{h}}\right)} \mathrm{e}^{-k_{\gamma} t}\right\}
\end{gathered}
$$

and

$$
\begin{aligned}
\frac{\left[\mathrm{I}^{*}(t)\right]}{\left[\mathrm{A}_{\mathrm{h}}^{*}(0)\right]}= & \frac{k_{\mathrm{h}} k_{\beta}+2 k_{\mathrm{r}} k_{\mathrm{p}}}{k_{\beta}\left(k_{\mathrm{I}}-k_{\gamma}\right)} \mathrm{e}^{-k_{\gamma} t} \\
& +\left\{\frac{2 k_{\mathrm{r}} k_{\mathrm{p}}-k_{\mathrm{h}}\left(k_{\mathrm{I}}-k_{\alpha}\right)}{\left(k_{\mathrm{I}}-k_{\alpha}\right)\left(k_{\mathrm{I}}-k_{\gamma}\right)}+\frac{\left[\mathrm{I}^{*}(0)\right]}{\left[\mathrm{A}_{\mathrm{h}}^{*}(0)\right]}\right\} \mathrm{e}^{-k_{\mathrm{I}} t} \\
& -\frac{2 k_{\mathrm{r}} k_{\mathrm{p}}}{k_{\beta}\left(k_{\mathrm{I}}-k_{\alpha}\right)} \mathrm{e}^{-k_{\alpha} t}
\end{aligned}
$$

where the effective rate coefficients $k_{\alpha}=k_{\mathrm{p}}+k_{\mathrm{T}}+k_{\mathrm{r}}$, $k_{\beta}=k_{\mathrm{p}}-k_{\mathrm{r}}-k_{\mathrm{h}}$, and $k_{\gamma}=k_{\mathrm{h}}+k_{\mathrm{T}}+2 k_{\mathrm{r}}$ were used for abbreviation. Note, that the amount of cross-well excitation can

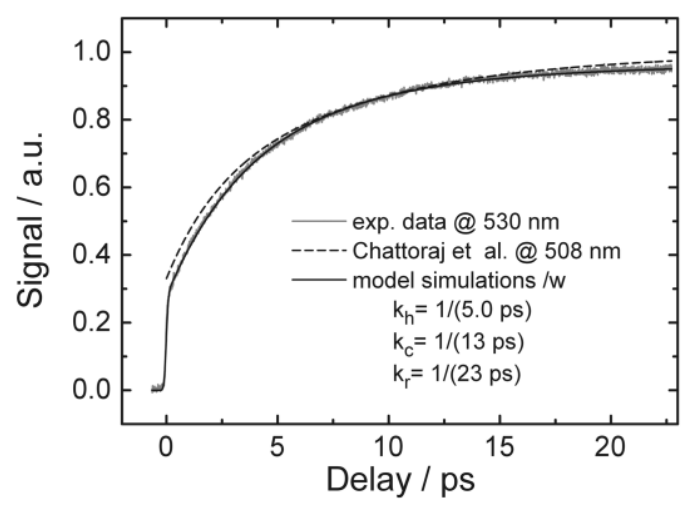

Fig. 8 Model simulation (black solid curve) of a pump-probe transient at $530 \mathrm{~nm}$ (gray curve) corresponding to the time-dependent population of I*. For comparison, the fluorescence upconversion data from ref. 11 are also reproduced (dashed curve). The rate constants are given in the legend.

be specified by using the boundary condition $\left[\mathrm{I}^{*}(0)\right]=1-\left[\mathrm{A}_{\mathrm{h}}^{*}(0)\right]$.

Since the excess energy is rather low, the vibrational species of $\mathrm{A}^{*}$ are spectroscopically indistinguishable. Neither can their products I* be resolved individually. Hence, $A^{*}$ is observed as the sum of over all populations $A_{h}^{*}+A_{p}^{*}+A_{c}^{*}$. To reduce the number of free adjustable parameters, we take $k_{\mathrm{I}}=1 / 4.2 \mathrm{~ns}$ and $k_{\mathrm{T}}=1 / 0.3 \mathrm{~ns}$ as determined from fluorescence lifetime measurements (see refs. 13,14,26,30,31). Furthermore, the extent of cross-well excitation is estimated from upconversion experiments reported by Boxer and coworkers. ${ }^{11}$ Hence, the ratio $\left[\mathrm{I}^{*}(0)\right] /\left[\mathrm{A}_{\mathrm{h}}^{*}(0)\right]$ is equal to 0.74 leaving $k_{\mathrm{r}}, k_{\mathrm{h}}$, and $k_{\mathrm{c}}$ as the only floating parameters to reproduce the pump-probe transients and the transient differential transmission spectra. In addition, the model has to reproduce the existing fluorescence lifetime and upconversion data reported in the literature as well as the quantum yield for emission as reported by Michel-Beyerle and coworkers. ${ }^{15}$ The latter quantity can be estimated from the above expressions according to

$$
\Phi_{\mathrm{f}}=1-\left[\mathrm{A}_{\mathrm{h}}^{*}(0)\right]\left\{1-\frac{2 k_{\mathrm{r}} k_{\mathrm{p}}-k_{\mathrm{h}}\left(k_{\mathrm{I}}-k_{\alpha}\right)}{\left(k_{\mathrm{I}}-k_{\alpha}\right)\left(k_{\mathrm{I}}-k_{\gamma}\right)}\right\}
$$

On the red edge of the stationary emission spectrum of GFP, it is possible to exclusively probe the dynamics of I* formation. This is because a probe wavelength of $530 \mathrm{~nm}$ circumvents

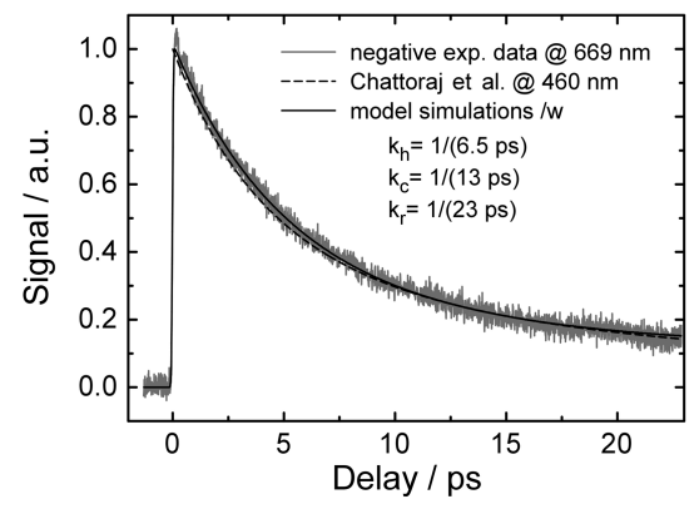

Fig. 9 Model simulation (black solid curve) of a pump-probe transient at $669 \mathrm{~nm}$ (gray curve) corresponding to the time-dependent population of $\mathrm{A}^{*}$. For comparison, the fluorescence upconversion data from ref. 11 are also reproduced (dashed curve). The rate constants are given in the legend. 


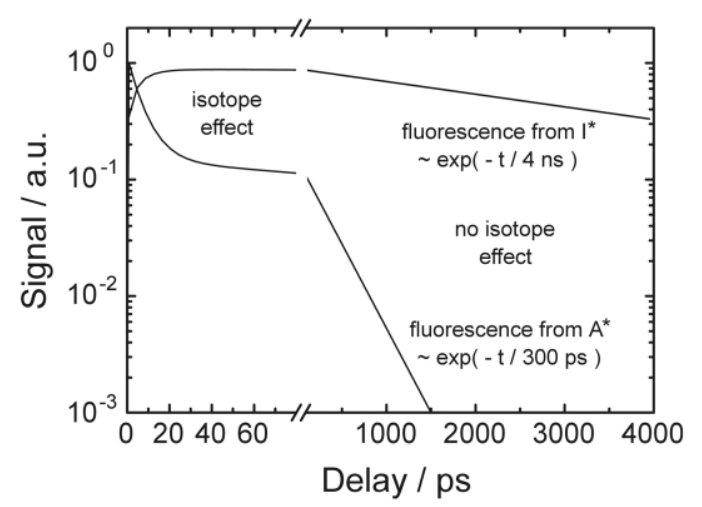

Fig. 10 Model simulation for the time-dependent populations of I* and $\mathrm{A}^{*}$ on picosecond and nanosecond time scales using the same rate parameters as given in Fig. 9. These simulations can be compared with corresponding fluorescence lifetime measurements as reported by Michel-Beyerle and coworkers. ${ }^{13,15,16}$ The model correctly predicts a slowly decaying component of $\mathrm{A}^{*}$ (corresponding to a time constant $1 / k_{\mathrm{T}}$ ) which lacks a kinetic isotope effect and does not have a counterpart in the build-up of I*.

contributions from the transient absorption observed at $\lambda=630 \mathrm{~nm}$ while simultaneously avoiding leakage from the transient absorption centered at $500 \mathrm{~nm}$. A comparison of the build-up of the stimulated emission at $530 \mathrm{~nm}$ with a model simulation for I* formation is shown in Fig. 8. The energy-specific rate coefficients, $k_{\mathrm{h}}$ and $k_{\mathrm{p}}$, were $0.2 \mathrm{ps}^{-1}$ and $0.077 \mathrm{ps}^{-1}$, respectively, whereas the rate coefficient for vibrational relaxation, $k_{\mathrm{r}}$, was set to $0.043 \mathrm{ps}^{-1}$. The agreement between simulation and experiment is excellent. Furthermore, the model is also in reasonable agreement with the fluorescence upconversion data from ref. 11. As discussed above, the A* decay can be monitored in transient absorption for probe wavelengths longer than $630 \mathrm{~nm}$. A model simulation for A* is reproduced in Fig. 9. Again, the agreement between simulation and experiment is excellent.

From the rate coefficients given in Fig. 9 together with eqn. (7), one obtains a quantum yield for internal conversion of $11 \%$, in good agreement with the value reported in ref. 15 . The long-time behavior of the $\mathrm{A}^{*}$ and $\mathrm{I}^{*}$ populations is reproduced in Fig. 10. Evidently, the model predicts I* to decay with a time constant of $1 / k_{\mathrm{I}}=4.2 \mathrm{~ns}$ whereas the long-time tail of the $\mathrm{A}^{*}$ decay is dictated by the time constant $1 / k_{\mathrm{T}}=0.3 \mathrm{~ns}$. Furthermore, the latter component is entirely missing in the rise of I*. Hence, the model quantitatively reproduces the streak camera fluorescence lifetime measurements from refs. $13,15,16$ including the peculiar isotope effect on short and long time scales as indicated in Fig. 10. This is because $k_{\mathrm{I}}$ and $k_{\mathrm{T}}$ are not related to proton motion as opposed to the rate coefficients $k_{\alpha}, k_{\beta}$, and $k_{\gamma}$ which define the early time behavior. Finally, one can simulate the transient differential transmission spectra, which are given by

$$
\varepsilon(t, \lambda)=\left\{\varepsilon_{\mathrm{E}}(\lambda)+\varepsilon_{\mathrm{TA}}(\lambda)\right\}\left[\mathrm{I}^{*}(t)\right]+\varepsilon_{\mathrm{A}}(\lambda)\left[\mathrm{A}^{*}(t)\right]
$$

Hence, the emission spectrum, $\varepsilon_{\mathrm{A}}$, of $\mathrm{A}^{*}$ is required. A good estimate for this quantity can be obtained from the mutant, P4-3, in which ESPT is effectively inhibited by replacement of the potential proton donor tyrosine at position 66 with a histidine residue. ${ }^{1}$ As shown in Fig. 11, its emission has a full width at half maximum of $3800 \mathrm{~cm}^{-1}$ and peaks around 449 $\mathrm{nm}$. In this wavelength range, a very weak but pronounced shoulder can be observed in the fluorescence spectrum of the wild-type protein, indicating that this feature originates primarily from $\mathrm{A}^{*}$. By shifting the mutant spectrum by 1000 $\mathrm{cm}^{-1}$ to lower frequencies, the blue edge of the wild type emission can be quantitatively reproduced (see Fig. 11). Therefore, this red-shifted mutant spectrum is taken as a rough estimate
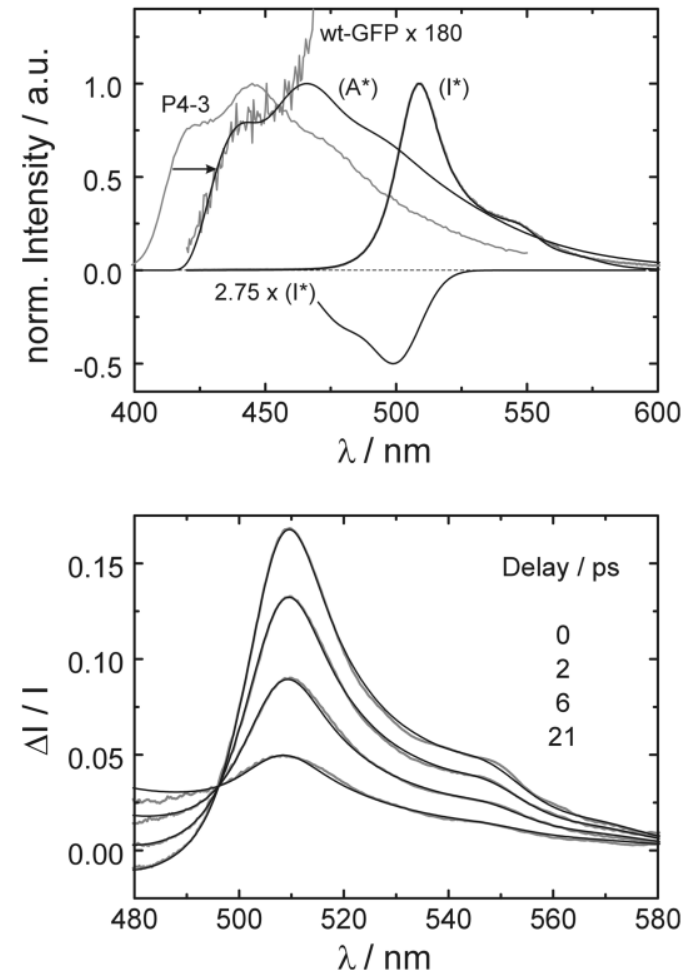

Fig. 11 Lower panel: Model simulation (black solid curve) of the transient differential transmission spectra (gray curves). The model correctly reproduces an isosbestic behavior around $496 \mathrm{~nm}$. The pump-WLC-probe time delays are indicated in the legend. Upper panel: Individual emission and transient absorption spectra for the $\mathrm{A}^{*}$ and $\mathrm{I}^{*}$ species which were used as input for the model simulations. The A* emission was estimated by shifting the steady-state spectra of the mutant P4-3 (BFP) by $1000 \mathrm{~cm}^{-1}$ to lower frequencies. This reproduces quantitatively the far blue edge of the steady-state spectrum of the wt-protein, which is reminiscent of the $A^{*}$ form of the wt-GFP.

for the $A^{*}$ emission. Its spectral profile can be represented in a numerically convenient format by

$$
\begin{aligned}
\varepsilon_{\mathrm{A}}(\tilde{v})= & \sum_{i} \varepsilon_{i}^{0} \Theta\left[\left(\tilde{v}_{i}-\Delta_{i} / 2 b_{i}\right)-\tilde{v}\right] \\
& \times \exp \left\{-\ln (2)\left[\frac{\ln \left(1+2 b_{i}\left(\tilde{v}-\tilde{v}_{i}\right) / \Delta_{i}\right)}{b_{i}}\right]^{2}\right\}
\end{aligned}
$$

where $\tilde{v}=1 / \lambda$ and $\Theta(\tilde{v})$ denotes the Heaviside step function. A sum over individual log-normal distributions, $i$, is used to reproduce the Franck-Condon fine structure typical for this mutant. The broadening and asymmetry parameters, $\Delta$ and $b$, of $1850 \mathrm{~cm}^{-1}$ and -0.49 have been used equally for all contributions. Their central wavelengths, $1 / \tilde{v}_{i}$, are $442.5,472.6$, 506.8 , and $540.5 \mathrm{~nm}$ with relative amplitudes, $\varepsilon_{i}$, of 45.6 , $36.8,14.6$, and $2.9 \%$.

Representative spectra and the corresponding model simulations are shown in the lower panel of Fig. 11 using the same set of rate coefficients as before. For comparison, the pure $\mathrm{A}^{*}$ and $I^{*}$ emissions as well as the I* transient absorption at $500 \mathrm{~nm}$ are also reproduced in the upper panel of Fig. 11. Again, a perfect agreement with the model simulations is found. Small discrepancies can only be seen at early delay times near the far blue edge of our detection window. These deviations may be due to the group velocity dispersion of the WLC probe pulses which have not been taken into account. The global agreement between the model simulations and a wide variety of experimental findings strongly supports a relaxation mechanism for the excited-state of GFP that includes convoluted time scales for reactive proton transfer and non-reactive inter and intramolecular vibrational energy transfer as well as an additional deactivation pathway leading to internal conversion via torsional motion of the chromophore. 


\section{E. Vibrational dynamics in the protein nanospace}

Within the framework of our model, inter and intramolecular vibrational energy relaxation occurs with a time constant of roughly $20 \mathrm{ps}$ and is slower by a factor of two compared to ESPT. The initial excess energy, despite being rather small, is similar to the threshold for ESPT, resulting in non-exponential dynamics. If vibrational relaxation were faster, the predominant mechanism for excited-state decay would be torsional motion with subsequent internal conversion. Therefore, a slow energy transfer in comparison to proton transfer is essential for efficient fluorescence from GFP. Fulfilment of this condition is facilitated by the relatively rigid protein shell that effectively protects the chromophore from inelastic collisions with environmental solvent particles. Opening the microenvironment exposes the chromophore to efficient energy dissipation and hence to quenching of fluorescence. Indeed, for the excited state of the photoactive yellow protein (PYP, phototactic receptor of eubacteria) whose chromophore, $p$-coumaric acid, is very similar to GFP but anchored to a highly solvent accessible region, cis-trans isomerization is the predominant relaxation pathway, ultimately triggering large amplitude motions in the protein backbone. ${ }^{33-35}$

Vibrational energy flow within $\beta$-barrel proteins and from such proteins to the surrounding solvent has been studied neither experimentally nor theoretically. Most of the experimental efforts on VET and IVR processes in proteic environments have been devoted to the amide-I region of small globular peptides. ${ }^{36}$ Vibrational relaxation was found to occur with a time constant of about 1.2 ps and was shown to be dominated by IVR, i.e by coupling of the amide-I mode to other vibrational motions of the protein backbone. ${ }^{36}$ Hence, such experiments are not really comparable to the vibrational energy flow described in the preceding sections. More relevant are experiments in which vibrational energy is initially deposited in the protein via optical excitation of its cofactor. For example, Lian et al. measured the dynamics of vibrational heating of solvent water following femtosecond optical excitation of the heme electronic states of myoglobin. ${ }^{37}$ The dissipation of excess energy from the heme into the protein, and later into its aqueous surroundings was found to take place on time scales of about 15 ps more similar to the time constant observed here. However, Xie et al. compared vibrational relaxation times of proteins with different secondary structure content. ${ }^{38}$ Again using amide-I excitation, they showed that proteins which consist mostly of $\alpha$-helices exhibit long-lived vibrationally excited states connected with a chain of hydrogen bonds connecting across the protein. ${ }^{37}$ This result seems to imply that such long relaxation times are characteristic for $\alpha$ helix proteins alone whereas in other proteins of predominantly $\beta$-sheet, such as GFP and PYP, such bottleneck states cannot exist. Indeed, the amide-I lifetime of PYP is again one order of magnitude shorter. Recent molecular dynamics simulations of energy transfer in proteins seem to indicate that the flow of vibrational excitation is governed by a high degree of mode specificity. ${ }^{39}$ Energy is transferred from a given normal mode of the protein to only a very few others, selected primarily by Fermi resonances. It is quite possible that such mode-specific couplings in combination with the chromophore's spatial isolation from the surrounding aqueous solution dictate the flow of energy from the GFP chromophore to its backbone and the rather slow vibrational relaxation rates in comparison to ESPT.

\section{Conclusions}

We have presented time and frequency resolved femtosecond pump-probe experiments to explore the elementary dynamics leading to fluorescence in the green fluorescent protein. In par- ticular, we have proposed a refined dynamic model for the primary events following ultrafast optical excitation. Besides excited-state deprotonation of the chromophore, the model includes vibrational relaxation as well as motion along a torsional coordinate preceding internal conversion as a secondary deactivation pathway. A wealth of spectroscopic results can be explained with this model provided vibrational relaxation of the initially prepared neutral chromophore occurs on time scales similar to the ESPT event. In this instance, channel switching becomes important such that at low excess energies internal conversion dominates. However, an upper limit for the quantum yield of this secondary pathway is defined by cross-well excitation, i.e. a significant fraction of the chromophores can be excited to the deprotonated form directly from the ground state neutral and will therefore remain emissive. The non-exponential kinetics observed in time resolved data are traced back to the competition between ESPT and vibrational relaxation. However, we strongly emphasize that the proposed model does not preclude the importance of structural heterogeneities of the hydrogen-bonded network to which the chromophore is coupled. In fact, the model can easily be extended in a phenomenological fashion to capture such inhomogeneous contributions to the reactive dynamics.

\section{Acknowledgement}

We gratefully acknowledge financial support by the Deutsche Forschungsgemeinschaft through the Sonderforschungsbereich 357 "Molekulare Mechanismen Unimolekularer Prozesse".

\section{References}

1 R. Y. Tsien, Annu. Rev. Biochem., 1998, 67, 509.

2 Green Fluorescent Protein: Properties, Applications and Protocols, ed. M. Chalfie and S. Kain, Wiley-Liss, New York, 1998.

3 A. B. Cubitt, R. Heim, S. R. Adams, A. E. Boyd, L. A. Gross and R. Y. Tsien, Trends Biochem. Sci., 1995, 20, 448.

4 M. Ormö, A. B. Cubitt, K. Kallio, L. A. Gross, R. Y. Tsien and S. J. Remington, Science, 1996, 273, 1392.

5 F. Yang, L. G. Moss and G. N. J. Phillips, Nature Biotech., 1996 14, 1246 .

6 K. Brejc, T. K. Sixma, P. A. Kitts, S. R. Kain, R. Y. Tsien, M. Ormö and S. J. Remington, Proc. Natl. Acad. Sci. USA, 1997, 94, 2306.

7 W. W. Ward, H. J. Prentice, A. F. Roth, C. W. Cody and S. C. Reeves, Photochem. Photobiol., 1982, 35, 803.

8 M. Chalfie, Y. Tu, G. Euskirchen, W. W. Ward and D. C. Prasher, Science, 1994, 263, 802

9 W. W. Ward, C. W. Cody, R. C. Hart and M. J. Cormier, Photochem. Photobiol., 1980, 31, 611 .

10 B. G. Reid and G. C. Flynn, Biochemistry, 1997, 36, 6786.

11 M. Chattoraj, B. A. King, G. U. Bublitz and S. G. Boxer, Proc. Natl. Acad. Sci. USA, 1996, 93, 8362.

12 D. C. Youvan and M. E. Michel-Beyerle, Nature Biotech., 1996, 14, 1219.

13 H. Lossau, A. Kummer, R. Heinecke, F. Pöllinger-Dammer, C. Kompa, G. Bieser, T. Jonsson, C. M. Silva, M. M. Yang, D. C. Youvan and M. E. Michel-Beyerle, Chem. Phys., 1996, 213, 1.

14 A. A. Voityuk, M. E. Michel-Beyerle and N. Rösch, Chem. Phys. Lett., 1997, 272, 162.

15 A. D. Kummer, C. Kompa, H. Lossau, F. Pöllinger-Dammer, M. E. Michel-Beyerle, C. M. Silva, E. J. Bylina, W. J. Coleman, M M. Yang and D. C. Youvan, Chem. Phys., 1998, 237, 183.

16 A. D. Kummer, J. Wiehler, H. Rehaber, C. Kompa, B. Steipe and M. E. Michel-Beyerle, J. Phys. Chem. B, 2000, 104, 4791.

17 T. M. H. Creemers, A. J. Lock, V. Subramaniam, T. M. Jovin and S. Völker, Nature Struct. Biol., 1999, 6, 557.

18 K. Mauring, A. Suisalu and J. Kikas, J. Lumin., 2000, 87-9, 812

19 T. M. H. Creemers, A. J. Lock, V. Subramaniam, T. M. Jovin and S. Völker, Proc. Natl. Acad. Sci. USA, 2000, 97, 2974.

20 G. Jung, C. Bräuchle and A. Zumbusch, J. Chem. Phys., 2001, 114, 3149 . 
21 G. Jung, J. Wiehler, W. Gohde, J. Tittel, T. Basché, B. Steipe and C. Bräuchle, Bioimaging, 1998, 6, 54

22 R. M. Dickson, A. B. Cubitt, R. Y. Tsien and W. E. Moerner, Nature, 1997, 388, 355.

23 M. F. Garcia-Parajo, G. M. J. Segers-Nolten, J. A. Veerman, J. Greve and N. F. van Hulst, Proc. Natl. Acad. Sci. USA, 2000, 97, 7237.

24 R. A. G. Cinelli, V. Tozzini, V. Pellegrini, F. Beltram, G. Cerullo, M. Zavelani-Rossi, S. De Silvestri, M. Tyagi and M. Giacca, Phys. Rev. Lett., 2001, 86, 3439.

25 K. Winkler, J. Lindner, H. Bürsing and P. Vöhringer, J. Chem. Phys., 2000, 113, 4674.

26 G. Striker, V. Subramaniam, C. A. M. Seidel and A. Volkmer, J. Phys. Chem. B, 1999, 103, 8612

27 R. Kubo, M. Toda and N. Hashitsume, Nonequilibrium Statistical Mechanics, Springer Verlag, Berlin, 1978.

28 J. Jethwa, D. Ouw, K. Winkler, N. Hartmann and P. Vöhringer, Z. Phys. Chem., 2000, 214, 1367.

29 A. L. Sobolewski and W. Domcke, Phys. Chem. Chem. Phys., 1999, 1, 3065.

30 A. A. Voityuk, M. E. Michel-Beyerle and N. Rösch, Chem. Phys. Lett., 1998, 296, 269.
31 A. Volkmer, V. Subramaniam, D. J. S. Birch and T. M. Jovin, Biophys. J., 2000, 78, 1589.

32 C. Xu, W. Zipfel, J. B. Shear, R. M. Williams and W. W. Webb, Proc. Natl. Acad. Sci. USA, 1996, 93, 10763.

33 W. D. Hoff, I. H. M. van Stokkum, H. J. van Ramesdonk, M. E. van Brederode, A. M. Brouwer, J. C. Fitch, T. E. Meyer, R. van Grondelle and K. J. Hellingwerf, Biophys. J., 1994, 67, 1691.

34 H. Chosrowjan, N. Mataga, N. Nakashima, Y. Imamoto and F. Tokunaga, Chem. Phys. Lett., 1997, 270, 267.

35 H. Chosrowjan, N. Mataga, Y. Shibata, Y. Imamoto and F. Tokunaga, J. Phys. Chem. B, 1998, 102, 7695.

36 P. Hamm, M. H. Lim and R. M. Hochstrasser, J. Phys. Chem. B, 1998, 102, 6123.

37 T. Q. Lian, B. Locke, Y. Kholodenko and R. M. Hochstrasser, $J$. Phys. Chem., 1994, 98, 11648.

38 A. H. Xie, L. van der Meer, W. Hoff and R. H. Austin, Phys. Rev. Lett., 2000, 84, 5435.

39 K. Moritsugu, O. Miyashita and A. Kidera, Phys. Rev. Lett., $2000,85,3970$ 